\title{
A TERMINAL COMPARISON PRINCIPLE FOR DIFFERENTIAL INEQUALITIES ${ }^{1}$
}

\author{
BY THOMAS G. HALLAM
}

Communicated by Fred Brauer, October 8, 1971

1. Introduction. The comparison principle has proved to be very useful in the study of various qualitative problems in ordinary differential equations. Comparison principles have been previously formulated in terms of initial value problems and, in this setting, their applications are numerous [6]. In this announcement, a comparison principle for terminal value problems is given. Related topics and applications are also discussed.

A comparison principle for terminal value problems has been stated in [7]; however, the proof given there for the weak inequality case needs some modification. In fact, the validity of a terminal comparison principle in the full generality of the statement in [7] remains an open question.

2. Preliminary hypotheses and definitions. A solution of the initial value problem which consists of the differential equation

$$
d r / d t=g(t, r)
$$

and the point $\left(t_{0}, r_{0}\right)$ will be denoted by $r\left(t, t_{0}, r_{0}\right)$. In (1), it is assumed that $g \in C\left[R_{+} \times R, R\right]$. It will be tacitly assumed that given any $r_{0} \in R$, there exists a $t_{0} \in R_{+}$so that the solution $r\left(t, t_{0}, r_{0}\right)$ of $(1)$ exists on $\left[t_{0}, \infty\right)$ and $\lim _{t \rightarrow \infty} r\left(t, t_{0}, r_{0}\right)$ exists. A solution of the terminal value problem consisting of equation (1) and a terminal value $r_{\infty}$ will be denoted by $r\left(t ; r_{\infty}\right)$.

A solution $r_{m}\left(t ; r_{\infty}\right)$ is a (the) maximal solution of the terminal value problem $\left\{(1) ; r_{\infty}\right\}$ on the interval I provided any other solution $u\left(t ; r_{\infty}\right)$ of the terminal value problem $\left\{(1) ; r_{\infty}\right\}$ which is valid on $I$ satisfies the inequality

$$
u\left(t ; r_{\infty}\right) \leqq r_{m}\left(t ; r_{\infty}\right) \quad(t \in I) .
$$

A similar definition may be given for the minimal solution of a terminal value problem. Either of the above types of solutions will be referred to as an extremal solution of the terminal value problem.

For initial value problems, the hypothesis $g \in C\left[R_{+} \times R, R\right]$ is sufficient to insure the existence of maximal and minimal solutions $[6, \mathrm{p} .11]$. However, even if the continuity of $g$ in $t$ is extended to the interval $[0, \infty]$,

AMS 1970 subject classifications. Primary 34A40, 34C10.

Key words and phrases. Comparison principle, differential inequalities, maximal and minimal solution, terminal value problem, asymptotic equilibrium.

${ }_{1}$ This research was supported in part by the National Science Foundation under Grant GP-11543. 
then extremal solutions of terminal value problems need not exist. A topic which, as will be noted below, is closely related to the existence of extremal solutions of terminal value problems is that of asymptotic equilibrium. F. Brauer [1], [2] and A. Wintner [8], [9], [10] have studied the asymptotic equilibrium problem in some detail.

Because the concept of extremal solutions is a local property of a differential equation, it is convenient to localize the definition of asymptotic equilibrium. Equation (1) is locally in asymptotic equilibrium about $r_{\infty}$ provided there exists a positive number $\delta$ with the property that whenever $r_{0}$ satisfies the inequality $\left|r_{0}-r_{\infty}\right|<\delta$ then the terminal value problem $\left\{(1) ; r_{0}\right\}$ has a solution. Hence, (1) is in asymptotic equilibrium if it is locally in asymptotic equilibrium about $r_{\infty}$ for every $r_{\infty} \in R$.

\section{Results on extremal solutions and asymptotic equilibrium.}

THEOREM 1. Let (1) have unique solutions to (finite) initial value problems. If (1) is locally in asymptotic equilibrium about $r_{\infty}$ then the terminal value problem $\left\{(1) ; r_{\infty}\right\}$ possesses both extremal solutions.

An immediate consequence of the above theorem is the following:

COROLlaRY. Let (1) have unique solutions to initial value problems. If (1) is in asymptotic equilibrium, then all terminal value problems possess both extremal solutions.

Examples may be constructed to show that if extremal solutions of a terminal value problem exist then the differential equation need not be locally in asymptotic equilibrium about a point. However, if it is known that all terminal value problems, when they exist, have both extremal solutions then the equation must be in asymptotic equilibrium.

THEOREM 2. If all terminal value problems of (1) have both extremal solutions, then (1) is in asymptotic equilibrium.

4. A terminal comparison principle. The terminal value problem $\left\{(1) ; r_{\infty}\right\}$ will be said to be suitable for perturbations in $L^{1}\left(R_{+}\right)$provided for any $h \in L^{1}\left(R_{+}\right) \cap C\left[R_{+}, R\right]$ the differential equation

$$
d r / d t=g(t, r)+h(t)
$$

is locally in asymptotic equilibrium about $\dot{r}_{\infty}$. Some situations in which a differential equation is suitable for perturbations in $L^{1}\left(R_{+}\right)$include the following:

(i) If (1) is in asymptotic equilibrium and $g$ is nonnegative and nondecreasing in $r$ for each fixed $t \in R_{+}$, then any terminal value problem is suitable for perturbations in $L^{1}\left(R_{+}\right)$. This may be verified by using the Lemma in [4]. 
(ii) If (1) is in asymptotic equilibrium and is uniformly convergent in variation, then any terminal value problem is suitable for perturbations in $L^{1}\left(R_{+}\right)$. This result is contained in [3].

In the next result, $D^{*}$ denotes the upper right Dini derivate.

THEOREM 3. Suppose that initial value problems of (1) have unique solutions. Suppose that the terminal value problem $\left\{(1) ; r_{\infty}\right\}$ is suitable for perturbations in $L^{1}\left(R_{+}\right)$. Let $r_{m}\left(t ; r_{\infty}\right)$ be the maximal solution of the terminal value problem $\left\{(1) ; r_{\infty}\right\}$ and suppose that $r_{m}\left(t ; r_{\infty}\right)$ exists on $J_{0}=\left[t_{0}, \infty\right)$. If $v(t)$ is a continuous function which satisfies the inequality

$$
D^{*} v(t) \geqq g(t, v(t)) \quad\left(t \in J_{0}\right)
$$

and the terminal inequality

$$
v(\infty) \leqq r_{\infty},
$$

then

$$
v(t) \leqq r_{m}\left(t ; r_{\infty}\right) \quad\left(t \in J_{0}\right) .
$$

5. Applications. Let $\|\cdot\|$ denote some norm of $n$-dimensional Euclidean space, $R^{n}$. Suppose that for $x \in R^{n}, t \in R_{+}$,

$$
\|f(t, x)\| \leqq g(t,\|x\|)
$$

where $f \in C\left[R_{+} \times R^{n}, R^{n}\right], g \in C\left[R_{+} \times R, R_{+}\right]$, and $g(t, r)$ is nondecreasing in $r$ for each fixed $t \in R_{+}$. It will also be assumed that

$$
\int^{\infty} t^{\sigma+1} g(t, \lambda) d t<\infty
$$

for all $\lambda>0$ and some $\sigma>0$. It is known that all solutions of

$$
d x / d t=f(t, x)
$$

are convergent provided (3) holds (see [2], [4]). That is, given any solution $x(t)$ of (4), there exists a vector $x_{\infty}$ such that the error function

$$
\rho_{x}(t) \equiv\left\|x(t)-x_{\infty}\right\| \in L_{0}^{\infty}\left(R_{+}\right) .
$$

Here, $L_{0}^{\infty}\left(R^{+}\right)$designates the subspace of $L^{\infty}\left(R_{+}\right)$that consists of all functions $x \in L^{\infty}\left(R_{+}\right)$such that ess $\lim _{t \rightarrow \infty} x(t)=0$.

The next result is an extension of one in [5]. The proof in [5] utilizes estimates of Brauer [2]; here the terminal comparison principle is applied.

THEOREM 4. Let the functions $f$ and $g$ satisfy (2) and (3). For each constant $c$, suppose that initial value problems of $d r / d t=-g(t, r+c)$ have unique solutions. Then, for any solution $x=x(t)$ of $(4), t^{\sigma} \rho_{x}(t) \in L_{0}^{\infty}\left(R_{+}\right) \cap L^{p}\left(R_{+}\right)$ for all $p \geqq 1$. 
A weight function other than $t^{\sigma}$ may be used in (3) and an analogous result to Theorem 4 may be obtained.

Among other applications which can be obtained from the terminal comparison principle are terminal analogues of the Perron Uniqueness Theorem and standard integral inequalities.

\section{REFERENCES}

1. F. Brauer, Global behavior of solutions of ordinary differential equations, J. Math. Anal. Appl. 2 (1961), 145-158. MR 24 \# A284.

2. - Bounds for solutions of ordinary differential equations, Proc. Amer. Math. Soc. 14 (1963), 36-43. MR 26 \#397.

3. T. G. Hallam, Convergence of solutions of nonlinear differential equations, Ann. Mat. Pura Appl. (to appear)

4. T. G. Hallam and J. W. Heidel, The asymptotic manifolds of a perturbed linear system of differential equations, Trans. Amer. Math. Soc. 149 (1970), 233-241. MR 41 \# 2136.

5. T. G. Hallam and V. Lakshmikantham, Growth estimates for convergent solutions of ordinary differential equations, J. Math. Phys. Sci. (to appear).

6. V. Lakshmikantham and S. Leela, Differential and integral inequalities. Theory and applications. Vol. I, Academic Press, New York, 1969.

7. Ja. D. Mamedov, One-sided estimates in the conditions for existence and uniqueness of solutions of the limit Cauchy problem in a Banach space, Sibirsk. Mat. Ž. 6 (1965), 1190-1196 (Russian). MR 32 \# 7908.

8. A. Wintner, Asymptotic equilibria, Amer. J. Math. 68 (1946), 125-132. MR 7, 297.

9. ㄴ An Abelian lemma concerning asymptotic equilibria, Amer. J. Math. 68 (1946), 451-454. MR 8, 71.

10. Amer. J. Math. 76 (1954), 183-190. MR 15, 426.

Department of Mathematics, Florida State University, Tallahassee, Florida 32306 